\title{
Longitudinal patterns of psychological distress in adult survivors of childhood cancer
}

\author{
T M Brinkman ${ }^{\star}, 1$, L Zhu ${ }^{2}$, L K Zeltzer ${ }^{3}$, C J Recklitis ${ }^{4}$, C Kimberg ${ }^{1}$, N Zhang ${ }^{2}$, A C Muriel ${ }^{5}$, M Stovall ${ }^{6}$, \\ D K Srivastava ${ }^{2}$, L L Robison ${ }^{1}$ and K R Krull ${ }^{1}$ \\ ${ }^{1}$ Department of Epidemiology and Cancer Control, St Jude Children's Research Hospital, 262 Danny Thomas Place, Memphis, TN \\ 38105, USA; ${ }^{2}$ Department of Biostatistics, St Jude Children's Research Hospital, 262 Danny Thomas Place, Memphis, TN 38105, USA; \\ ${ }^{3}$ UCLA Department of Pediatrics, David Geffen School of Medicine at UCLA, Box 951752, Los Angeles, CA 90095, USA; ${ }^{4}$ Perini Family \\ Survivor's Center, Dana-Farber Cancer Institute, 450 Brookline Avenue, Boston, MA 02215, USA; ${ }^{5}$ Department of Psychosocial \\ Oncology and Palliative Care, Dana-Farber Cancer Institute, 450 Brookline Avenue, Boston, MA 02215, USA and ${ }^{6}$ Department of \\ Radiation Physics, University of Texas MD Anderson Cancer Center, 1515 Holcombe Boulevard, Houston, TX 77030, USA
}

Background: This study investigated longitudinal patterns of psychological distress in adult survivors of childhood cancer.

Methods: Participants included 4569 adult survivors in the Childhood Cancer Survivor Study Cohort (CCSS) who completed the Brief Symptom Inventory-18 on three occasions between 1994 and 2010. Longitudinal latent class analysis was used to identify discrete classes of psychological distress. Predictors of class membership were examined through logistic regression modelling with odds ratios (ORs) and 95\% confidence intervals (Cls) reported.

Results: Survivors were a median of 39 years of age and 30 years from diagnosis at the most recent follow-up. Most survivors reported few or no symptoms of distress over time, although subsets of survivors reported persistently elevated (depression: 8.9\%; anxiety: $4.8 \%$; somatisation: $7.2 \%$ ) or significant increases in distress symptoms over the follow-up period (depression: 10.2\%; anxiety: $11.8 \%$; somatisation: $13.0 \%$ ). Increasing distress symptoms were predicted by survivor perception of worsening physical health over time (depression: $\mathrm{OR}=3.3 ; 95 \% \mathrm{Cl}=2.4-4.5$; anxiety: $\mathrm{OR}=3.0 ; 95 \% \mathrm{Cl}=2.2-4.0$; somatisation: $\mathrm{OR}=5.3 ; 95 \% \mathrm{Cl}=3.9-7.4$ ). Persistent distress symptoms were also predicted by survivor perception of worsening physical health over time, as well as by worsening pain and ending analgesic use.

Conclusion: Subgroups of adult survivors are at-risk for chronic distress or significant increases in distress decades following their original cancer diagnosis. Routine screening of psychological distress in adult survivors of childhood cancer is warranted, especially for survivors who experience physical health morbidities.

Treatment advances leading to cure or longer survival for malignant diseases in childhood have resulted in over 360000 childhood cancer survivors in the United States (Howlader et al, 2011). Morbidity associated with increased survival often involves many organ systems (Hudson et al, 2003) and has the potential to adversely impact psychological functioning (Zeltzer et al, 2008; Michel et al, 2010). The consequences of distress are considerable with increased rates of suicide ideation documented among survivors with depressive symptoms (Recklitis et al, 2006a, 2010).
As fewer than $20 \%$ of long-term survivors receive follow-up care by an oncologist (Oeffinger et al, 2004), the responsibility of identifying psychological morbidities in this growing population often falls to primary-care providers.

Several long-term follow-up studies of childhood cancer survivors suggest that although most adult survivors are psychologically healthy, subgroups are at-risk for experiencing significant emotional distress, including symptoms of depression, anxiety and somatisation (Zebrack et al, 2004; Zeltzer et al, 2008; Michel et al, 2010). 
Specifically, survivors of central nervous system malignancies report higher levels of depression than comparison siblings and US population norms, while survivors of Hodgkin and non-Hodgkin lymphoma report greater somatic symptoms (Zebrack et al, 2004, 2007; Zeltzer et al, 2008). Risk factors for elevated distress symptoms include female sex, cranial irradiation, single or divorced marital status, unemployment, and annual household income $<\$ 20000$ (Zeltzer et al, 2008).

In the general population, greater levels of distress are present among adult females and individuals with lower compared with higher levels of educational attainment (Schieman et al, 2001). Recent longitudinal data suggest that these disparities, observed throughout adulthood, are a result of psychological distress specific to a group of individuals with persistent distress rather than acute distress among continually changing groups of individuals (Miech et al, 2007). However, psychological distress in adulthood also has been associated with changes in socioeconomic factors (e.g., job loss, divorce) and health status (Hope et al, 1999; Miech and Shanahan, 2000). How patterns of psychological distress persist or change among adult survivors of childhood cancer, who are vulnerable to declines in health status, are largely unknown.

The established risk factors of distress in survivors and the general population, lend support for a conceptual model of psychological functioning, which considers the dynamic interplay of biological, psychological and social factors (i.e., biopsychosocial framework; Engel, 1981). The primary aim of this study was to investigate longitudinal patterns of psychological distress in a large cohort of adult survivors of childhood cancer using a biopsychosocial framework. Although previous reports suggest that psychological distress affects only a subgroup of adult survivors, the cross-sectional nature of these studies has precluded understanding how psychological distress may change over the course of survivorship (Zebrack et al, 2004, 2007; Zeltzer et al, 2008). Importantly, a longitudinal approach allows for the identification of risk and protective factors that may influence the trajectory of psychological distress. Knowledge of how symptoms of psychological distress persist or change over time, as well as factors that influence such patterns, are essential to guide the development and timing of screening and interventions to address distress disparities among survivors. We hypothesised that female survivors would demonstrate increased risk of persistent distress and that adverse changes in socioeconomic factors (e.g., income, employment and marital status) would be associated with increased risk of persistent psychological distress. We also hypothesised that patterns of change in self-reported physical health status would be associated with patterns of change in psychological distress (i.e., improved physical health status will be associated with reduced psychological distress; worsened cancer-related pain will be associated with increased psychological distress).

\section{MATERIALS AND METHODS}

Childhood cancer survivor study (CCSS). The CCSS cohort consists of survivors of the most common forms of childhood cancer diagnosed at $\leqslant 21$ years of age and treated at one of 26 institutions between 1970 and 1986. All survivors were 5 or more years from their original diagnosis upon study enrolment. The study was approved by the institutional review board at each collaborating institution and informed consent was obtained from each study participant. Study participants completed a baseline questionnaire beginning in 1994 and subsequent follow-up questionnaires initiated in 2003 and 2007. Additional descriptions of the CCSS methodology and participants have been published elsewhere (Leisenring et al, 2009; Robison et al, 2009). Recruitment of this study population is shown in Figure 1 and included 4569

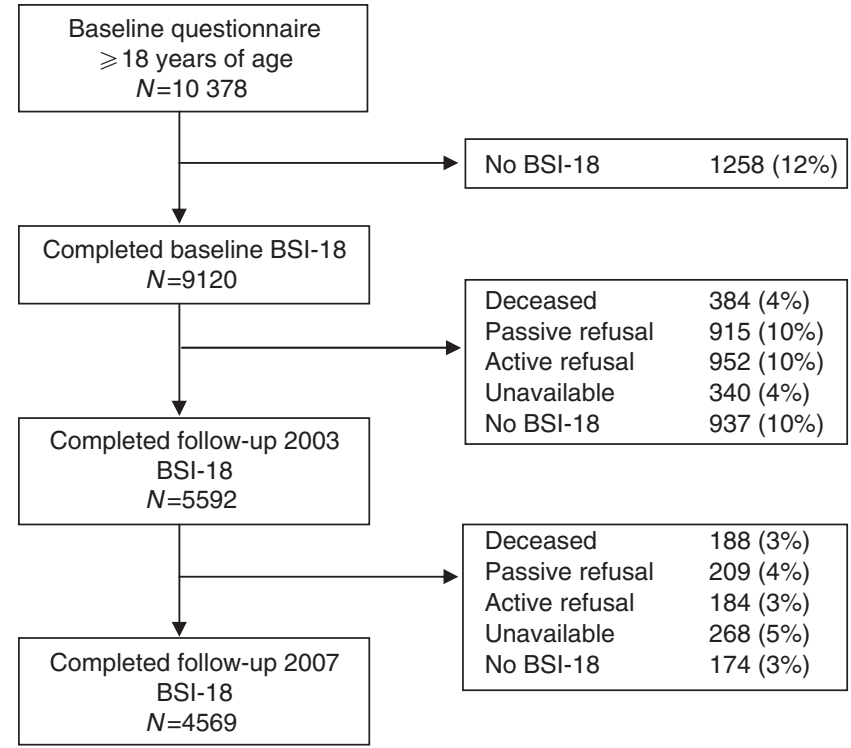

Figure 1. Consort diagram of study participation. BSI-18= Brief Symptom Inventory.

cancer survivors $\geqslant 18$ years of age at baseline who completed the Brief Symptom Inventory-18 (BSI-18) independently at baseline, follow-up 2 (2003-2005) and follow-up 3 (2007-2010).

Primary outcomes. Psychological distress was measured by the BSI-18 (Derogatis, 2000). The BSI-18 is an 18-item questionnaire that utilises a five-point Likert scale response format and provides a global measure of psychological distress as well as subscales for anxiety, depression and somatisation. Strong correlations between the BSI-18 and Symptom Checklist-90-Revised have been reported (Recklitis and Rodriguez, 2007) and the three-factor model of distress has been validated in adult survivors of childhood cancer (Recklitis et al, 2006b). In the current sample, Cronbach's alpha was calculated at each assessment point across subscales (baseline: depression $=0.88$, anxiety $=0.80$, somatisation $=0.72$; 2003 follow-up: depression $=0.89$, anxiety $=0.82$, somatisation $=$ $0.76 ; 2007$ follow-up: depression $=0.90, \quad$ anxiety $=0.82$, somatisation $=0.73$ ). Raw scores were converted to $\mathrm{T}$-scores using sex-specific normative data from a sample of community dwelling adults in the United States (Derogatis, 2000). Higher scores on the BSI represent greater levels of distress. Scores falling $\geqslant 90$ th percentile (i.e., T-score $\geqslant 63$ ) were classified as representing a clinical level of emotional distress.

Predictors and covariates. Demographic and socioeconomic variables considered in the analyses included sex, age at the time of survey completion, health insurance status, personal income $(<\$ 20000$ or $\geqslant \$ 20000)$, employment status (employed vs unemployed), educational attainment ( $\leqslant$ high school graduate or $\geqslant$ some college) and marital status (married/living as married, divorced/separated/widowed, or single/never married). Cancerrelated variables, measured at baseline, included age at diagnosis, radiation therapy and chemotherapy (yes/no). Radiation therapy was categorised as none, non-cranial and cranial radiation, which was further dichotomised as $\leqslant 20$ and $>20 \mathrm{~Gy}$. Survivor report of physical health status (fair or poor $v s$ good, very good, or excellent) and cancer-related pain (none or small amount vs medium amount, a lot or very bad) were included as primary predictors. Antidepressant, anxiolytic and analgesic medication use were included as covariates (for description of medication classification, see Brinkman et al, 2013). Survivors completed a 289-item survey that included questions regarding their physical health conditions, including age of onset of the condition. To determine severity of 
the conditions, scoring was based on the Common Terminology Criteria for Adverse events (version 3.0; Cancer Therapy Evaluation Program, 2003) was used to grade conditions as mild (grade 1) or moderate (grade 2) vs severe (grade 3) or lifethreatening/disabling (grade 4) at baseline (Oeffinger et al, 2006). This scoring system was developed through the National Cancer Institute and intended for use in scoring acute and chronic conditions in patients with cancers and survivors of all ages (Trotti et al, 2003). Unless otherwise noted, all demographic, socioeconomic, and health-related predictors and covariates were measured at baseline and follow-up 3. Cancer-related pain was measured at baseline and follow-up 2. Change in covariates and predictors over time were coded as no change $v s$ positive change (e.g., improved physical health status) or no change $v s$ negative change (e.g., worsened physical health status). For example, worsened physical health status was defined as report of good, very good or excellent health status at baseline and fair or poor health status at follow-up 3 while improved physical health status was defined as report of the reverse pattern. No change was defined as report of symptoms that fell into the same category at baseline and follow-up 3.

Statistical analysis. Descriptive statistics were calculated for all outcomes, predictors and covariates used in the analyses. We used a structural equation modelling (SEM) technique that allowed for the identification of discrete profiles of psychological distress (Mplus 6; Muthén and Muthén, 1998-2010). Longitudinal latent profile analysis (LPA), a submodel of SEM, is a multiple-group structural equation model in which the group variable is unobserved. LPA uses measured variables (e.g., psychological distress) to identify different groups of survivors where it is hypothesised that group differences exist but the number and nature of the groups is not known. The grouping variable (e.g., class membership) is not measured or observed but is derived from the observed data. In LPA, multiple statistical indicators are used to select the best fitting model. Fit indices considered in the current analysis included (1) Bayesian Information Criterion, with the lowest value among competing models indicating best fit; (2) entropy, an estimate of how well the model classifies subjects, with values closer to 1.0 indicating better classification; (3) Vuong-LoMendell-Rubin likelihood ratio test (VLMR) and sample sizeadjusted VLMR with $P$-values $<0.05$ indicating a significant difference in model fit between two nested models that vary by one class; and (4) minimum class membership $\geqslant 5 \%$ to provide sufficient power for subsequent analyses. Models were fit with two through six profiles for each distress index to determine the optimal number of profiles needed to describe survivors from baseline through follow-up 3. Posterior probability testing was also

\section{Table 1. Baseline characteristics for survivors with and without all BSI time points}

\begin{tabular}{|c|c|c|c|c|}
\hline & \multicolumn{2}{|c|}{ Survivors $\mathrm{BSI}=3^{\mathrm{a}}(\mathrm{N}=4569)$} & \multicolumn{2}{|c|}{ Survivors $\geqslant 1 \mathrm{BSI}<3^{\mathrm{b}}(\mathrm{N}=4890)$} \\
\hline & Mean (s.d.) & Range & Mean (s.d.) & Range \\
\hline $\begin{array}{l}\text { Age at diagnosis, years } \\
\text { Time since diagnosis, years } \\
\text { Baseline age, years }\end{array}$ & $\begin{array}{l}10.0(5.6) \\
17.4(4.6) \\
27.4(6.0)\end{array}$ & $\begin{array}{c}0-20 \\
6-32 \\
18-48\end{array}$ & $\begin{array}{r}9.1(5.6) \\
17.4(4.6) \\
26.5(6.2)\end{array}$ & $\begin{array}{c}0-20 \\
6-30 \\
18-47\end{array}$ \\
\hline \multicolumn{5}{|c|}{ Psychological distress at baseline } \\
\hline $\begin{array}{l}\text { Depression } \\
\text { Anxiety } \\
\text { Somatisation }\end{array}$ & $\begin{array}{l}47.9(8.9) \\
46.6(8.8) \\
47.5(7.6)\end{array}$ & $\begin{array}{l}40-81 \\
38-81 \\
41-81\end{array}$ & $\begin{array}{l}48.5(9.8) \\
46.6(9.5) \\
47.9(8.5)\end{array}$ & $\begin{array}{l}40-81 \\
38-81 \\
41-81\end{array}$ \\
\hline Somatisation & Frequency & $\%$ & Frequency & $\%$ \\
\hline \multicolumn{5}{|l|}{ Gender } \\
\hline $\begin{array}{l}\text { Male } \\
\text { Female }\end{array}$ & $\begin{array}{l}2227 \\
2342\end{array}$ & $\begin{array}{l}48.7 \\
51.3\end{array}$ & $\begin{array}{l}2774 \\
2116\end{array}$ & $\begin{array}{l}56.7 \\
43.3\end{array}$ \\
\hline \multicolumn{5}{|l|}{ Diagnosis } \\
\hline $\begin{array}{l}\text { Leukaemia } \\
\text { CNS tumour } \\
\text { Hodgkin lymphoma } \\
\text { Non-Hodgkin lymphoma } \\
\text { Wilms tumour } \\
\text { Neuroblastoma } \\
\text { Soft tissue sarcoma } \\
\text { Osteosarcoma }\end{array}$ & $\begin{array}{r}1354 \\
526 \\
882 \\
394 \\
278 \\
163 \\
460 \\
512\end{array}$ & $\begin{array}{c}29.6 \\
11.5 \\
19.3 \\
8.6 \\
6.1 \\
3.6 \\
10.1 \\
11.2\end{array}$ & $\begin{array}{r}1473 \\
655 \\
780 \\
468 \\
349 \\
224 \\
442 \\
499\end{array}$ & $\begin{array}{c}30.1 \\
13.4 \\
16.0 \\
9.6 \\
7.1 \\
4.6 \\
9.0 \\
10.2\end{array}$ \\
\hline \multicolumn{5}{|l|}{ Radiation therapy } \\
\hline $\begin{array}{l}\text { None } \\
\text { Non-cranial } \\
\text { CRT } \leqslant 20 \text { Gy } \\
\text { CRT }>20 \text { Gy }\end{array}$ & $\begin{array}{r}1203 \\
1463 \\
523 \\
1032\end{array}$ & $\begin{array}{l}28.5 \\
34.7 \\
12.4 \\
24.5\end{array}$ & $\begin{array}{r}1095 \\
1257 \\
507 \\
1000\end{array}$ & $\begin{array}{l}28.4 \\
32.6 \\
13.1 \\
25.9\end{array}$ \\
\hline $\begin{array}{l}\text { Abbreviations: BSI- } 18=\text { Brief Sym } \\
a_{\mathrm{BSI}}=3 \text { : completed all three que } \\
\mathbf{b}_{\geqslant} \geqslant 1 \mathrm{BSI}<3 \text { : completed one or } \mathrm{t}\end{array}$ & tral ner & ial rad & & \\
\hline
\end{tabular}


conducted to determine how well each participant fit into their assigned class.

Once class membership was established via the above methodology, predictors of class membership were examined through logistic regression modelling with robust variance estimates to account for within subject correlation using SAS version 9.2 PROC Logistic (SAS Institute, Cary, NC, USA). Multivariable models, considering all possible combinations of predictors and covariates, with the smallest Akaike information criterion were selected as the final model for each distress outcome. Odds ratios (ORs) and 95\% confidence intervals (CIs) were calculated for all predictors and covariates retained in the final model.

\section{RESULTS}

Characteristics of survivors who completed the BSI-18 at all three study time points were similar to those of survivors who completed the BSI-18 at only one or two time points (Table 1). A slightly larger proportion of females completed the BSI-18 at all three time points compared with males $(P \leqslant 0.001)$. Survivors who completed the BSI-18 at all three time points were, on average, 27 years of age at baseline (17 years from diagnosis), 35 years at follow-up 2 (25 years from diagnosis) and 40 years at follow-up 3 (30 years from diagnosis).

The LPA modelling indicated that survivors fell into four meaningful classes of depression (posterior probability range: 0.97-1.0, mean: 0.98), anxiety (posterior probability range: 0.85-1.0, mean: 0.89) and somatisation (posterior probability range: $0.92-0.98$, mean: 0.94 ) based on their symptom scores over time. Figure 2 depicts the four distinct longitudinal patterns of symptoms of depression (Figure 2A), anxiety (Figure 2B) and somatisation (Figure 2C) defined by our models: (class 1) survivors with few or no symptoms at all time points; (class 2) survivors with elevated symptoms at baseline that decreased over time; (class 3) survivors with few or no symptoms at baseline that increased over time; and (class 4) survivors with elevated symptoms that persisted over time. Supplementary online Table 1 provides the model fit indices for two to six class solutions for each distress outcome. Mean scores for each longitudinal class across all study time points are shown separately for depression, anxiety and somatisation (Table 2).

Persistent distress symptoms. For each psychological outcome, a subgroup of survivors was classified as reporting persistently elevated or chronic symptoms of distress (Table 2: class 4: depressive symptoms: $8.9 \%$; anxiety symptoms: $4.8 \%$; somatic symptoms: $7.2 \%$ of survivors). Compared with the majority of survivors who reported few to no symptoms of distress over time (class 1), class 4 membership was predicted by the presence of a mild-to-moderate medical condition at baseline (depression: $\mathrm{OR}=1.6 ; 95 \% \mathrm{CI}=1.2-2.2$; anxiety: $\mathrm{OR}=1.6 ; 95 \% \mathrm{CI}=1.1-2.5$; somatisation: $\mathrm{OR}=1.8 ; 95 \% \mathrm{CI}=1.2-2.9)$, perception of worsening physical health over time (depression: $\mathrm{OR}=2.9 ; 95 \% \mathrm{CI}=2.0-4.1$; anxiety: $\mathrm{OR}=3.4 ; 95 \% \mathrm{CI}=2.3-5.4$; somatisation: $\mathrm{OR}=4.4 ; 95 \%$ $\mathrm{CI}=2.8-6.8$ ) and increased cancer-related pain (depression: $\mathrm{OR}=2.1 ; \quad 95 \% \quad \mathrm{CI}=1.4-3.2 ; \quad$ somatisation: $\mathrm{OR}=3.3 ; \quad 95 \%$ $\mathrm{CI}=2.0-5.4)$. Change in marital status from previously being married to being single was associated with increased likelihood of persistent depressive symptoms $(\mathrm{OR}=2.3,95 \% \mathrm{CI}=1.1-4.6)$, whereas change in employment status to unemployed was associated with persistent somatic symptoms ( $\mathrm{OR}=1.8,95 \%$ $\mathrm{CI}=1.2-2.8$; see Table 3 ). Radiation therapy, employment change and personal income change did not contribute to models predicting persistent symptoms of anxiety or depression.
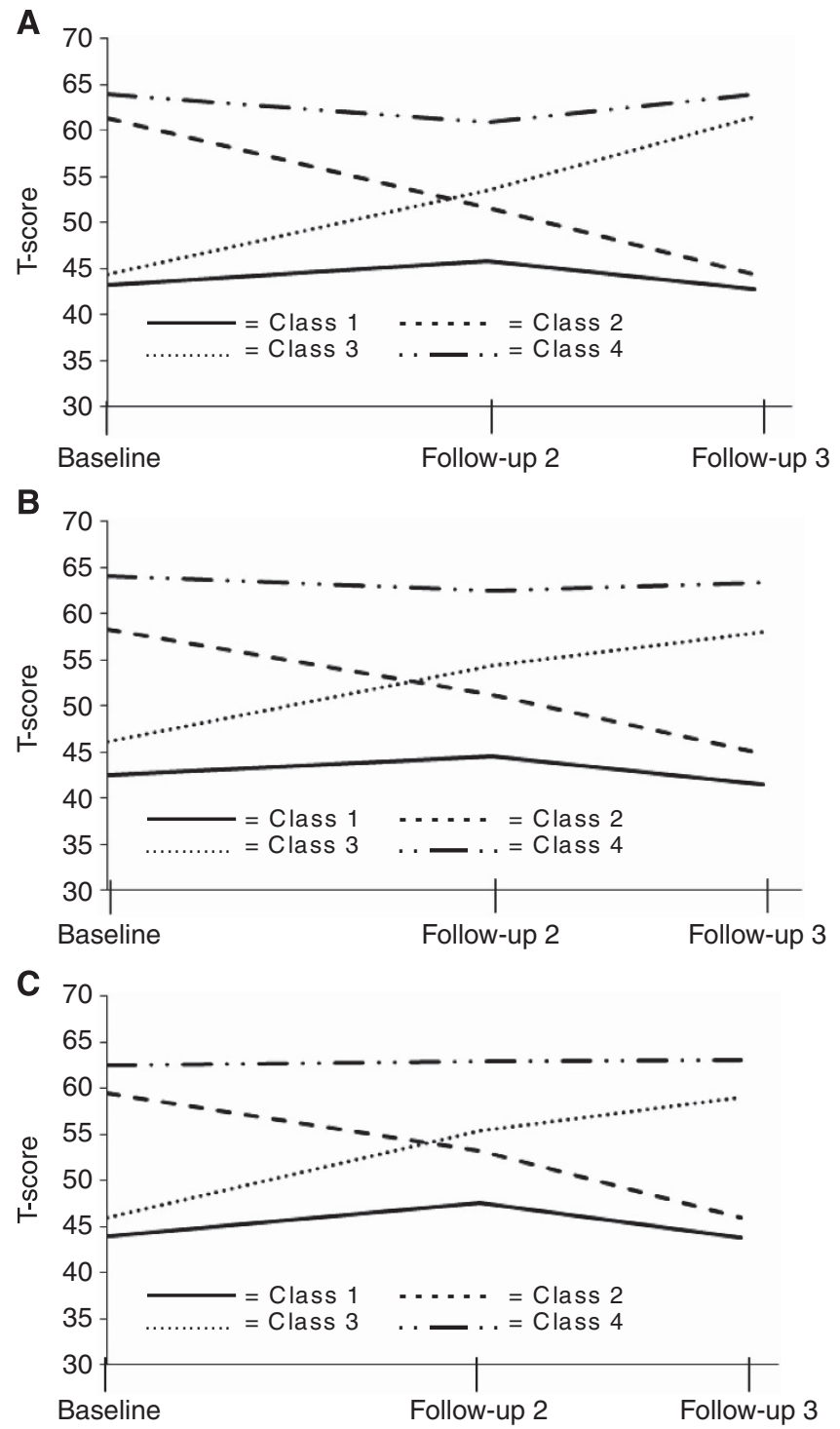

Figure 2. Longitudinal classes of distress symptoms across baseline and two follow-up time points. Vertical axis depicts average T-score (expected mean $=50$; s.d. $=10$ ) for each class at various time points. (A) Depressive symptoms. (B) Anxiety symptoms. (C) Somatic symptoms.

Increasing distress symptoms. Class 3 defined by our models comprises survivors who reported few or no symptoms at baseline with increasing symptoms of distress over time (depressive symptoms: $10.2 \%$; anxiety symptoms: $11.8 \%$; somatic symptoms: $13.0 \%$ of survivors), such that significant levels were observed within the group by the end of follow-up. Compared with survivors with few to no symptoms over time (class 1), class 3 membership across all symptoms was predicted by reported perception of worsening physical health over time (depression: $\mathrm{OR}=3.3 ; 95 \% \mathrm{CI}=2.4-4.5$; anxiety: $\mathrm{OR}=3.0 ; 95 \% \mathrm{CI}=2.2-4.0$; somatisation: $\mathrm{OR}=5.3 ; 95 \% \mathrm{CI}=3.9-7.4)$. Class 3 membership for symptoms of somatisation was associated with perception of increased cancer-related pain over time $(\mathrm{OR}=2.4 ; 95 \% \mathrm{CI}=1.6-3.6)$ and female sex $(O R=1.6,95 \% C I=1.3-2.0$; see Table 3$)$. Radiation therapy did not significantly contribute to multivariable models predicting increasing distress symptoms over time.

Decreasing distress symptoms. Among the identified classes of symptoms, class 2 and class 4 are characterised by elevated distress symptoms at baseline, with a reduction in symptoms to subclinical 


\begin{tabular}{|c|c|c|c|c|c|c|c|c|}
\hline \multirow[b]{2}{*}{ Class } & \multirow[b]{2}{*}{$\mathbf{N}$} & \multirow[b]{2}{*}{$\%$} & \multicolumn{2}{|c|}{ Baseline } & \multicolumn{2}{|c|}{2003} & \multicolumn{2}{|c|}{2007} \\
\hline & & & Mean (s.d.) & $\mathbf{N}(\% \operatorname{lmp})$ & Mean (s.d.) & $\mathbf{N}(\% \operatorname{Imp})$ & Mean (s.d.) & $\mathbf{N}(\% \operatorname{Imp})$ \\
\hline \multicolumn{9}{|c|}{ Depression } \\
\hline $\begin{array}{l}1 \\
2 \\
3 \\
4\end{array}$ & $\begin{array}{r}3005 \\
690 \\
466 \\
408\end{array}$ & $\begin{array}{r}65.8 \\
15.1 \\
10.2 \\
8.9\end{array}$ & $\begin{array}{l}43.3(3.3) \\
61.4(4.5) \\
44.2(3.6) \\
63.9(5.8)\end{array}$ & $\begin{aligned} 0 & (0.0) \\
217 & (31.5) \\
0 & (0.0) \\
192 & (47.1)\end{aligned}$ & $\begin{array}{l}45.9(7.0) \\
51.5(9.7) \\
53.5(10.4) \\
60.7(9.9)\end{array}$ & $\begin{array}{l}116(3.9) \\
100(14.5) \\
100(21.5) \\
180(44.1)\end{array}$ & $\begin{array}{l}42.8(3.0) \\
44.3(4.0) \\
61.4(4.8) \\
63.8(6.2)\end{array}$ & $\begin{array}{c}0(0.0) \\
0(0.0) \\
136(29.2) \\
186(45.6)\end{array}$ \\
\hline \multicolumn{9}{|c|}{ Anxiety } \\
\hline $\begin{array}{l}1 \\
2 \\
3 \\
4\end{array}$ & $\begin{array}{r}3123 \\
691 \\
537 \\
218\end{array}$ & $\begin{array}{r}68.4 \\
15.2 \\
11.8 \\
4.8\end{array}$ & $\begin{array}{l}42.5(4.9) \\
59.3(5.0) \\
46.1(5.7) \\
64.6(6.5)\end{array}$ & $\begin{aligned} 0 & (0.0) \\
149 & (21.6) \\
0 & (0.0) \\
123 & (56.4)\end{aligned}$ & $\begin{array}{l}44.5(6.6) \\
51.3(8.1) \\
54.9(9.1) \\
62.5(9.3)\end{array}$ & $\begin{array}{c}47(1.5) \\
55(8.0) \\
98(18.2) \\
109(50.0)\end{array}$ & $\begin{array}{l}41.4(4.4) \\
44.8(5.4) \\
58.6(5.6) \\
63.7(6.5)\end{array}$ & $\begin{array}{c}0(0.0) \\
0(0.0) \\
100(18.6) \\
102(46.8)\end{array}$ \\
\hline \multicolumn{9}{|c|}{ Somatisation } \\
\hline $\begin{array}{l}1 \\
2 \\
3 \\
4\end{array}$ & $\begin{array}{r}3114 \\
532 \\
592 \\
331\end{array}$ & $\begin{array}{r}68.2 \\
11.6 \\
13.0 \\
7.2\end{array}$ & $\begin{array}{l}44.0(3.5) \\
59.8(4.4) \\
46.1(4.6) \\
62.6(5.3)\end{array}$ & $\begin{array}{c}0(0.0) \\
150(28.2) \\
0(0.0) \\
165(49.8)\end{array}$ & $\begin{array}{l}47.7(7.3) \\
52.8(8.3) \\
55.2(8.8) \\
63.2(8.0)\end{array}$ & $\begin{aligned} 187 & (6.0) \\
81 & (15.2) \\
141 & (23.8) \\
201 & (60.7)\end{aligned}$ & $\begin{array}{l}43.8(3.5) \\
45.8(4.4) \\
59.4(4.0) \\
63.2(5.4)\end{array}$ & $\begin{array}{c}0(0.0) \\
0(0.0) \\
152(25.7) \\
194(58.6)\end{array}$ \\
\hline
\end{tabular}

levels observed over time for class 2 (depressive symptoms: $15.1 \%$; anxiety symptoms: $15.2 \%$; somatic symptoms: $11.6 \%$ of survivors) compared with persistent distress symptoms observed for class 4 . A reduction in anxiety symptoms (i.e., class 2 membership) was associated with decreased likelihood of worsened cancer-related pain $(\mathrm{OR}=0.5 ; 95 \% \mathrm{CI}=0.3-1.0)$, decreased likelihood of worsened physical health status $(\mathrm{OR}=0.4 ; 95 \% \mathrm{CI}=0.2-0.6)$ and increased likelihood of change in marital status from being single to married $(\mathrm{OR}=1.6 ; 95 \% \mathrm{CI}=1.0-2.5)$ relative to survivors in class 4 . A reduction in depressive symptoms was associated with decreased likelihood of worsened physical health status $(\mathrm{OR}=0.4$; $95 \% \mathrm{CI}=0.2-0.6)$ and decreased likelihood of change in employment status to unemployed $(\mathrm{OR}=0.7 ; 95 \% \mathrm{CI}=0.5-1.0)$. Survivors treated with non-cranial radiation were significantly less likely to report decreasing somatic symptoms (OR $=0.6 ; 95 \%$ $\mathrm{CI}=0.4-0.9)$. Survivors with worsened physical health status were also less likely to have decreasing somatic symptoms over time $(\mathrm{OR}=0.3 ; 95 \% \mathrm{CI}=0.2-0.5$; see Table 4). Chronic medical conditions were unrelated to decreasing symptoms of psychological distress.

\section{DISCUSSION}

We report on longitudinal patterns of psychological distress in a large cohort of adult survivors of childhood cancer. Our findings are novel and compelling because not only do we demonstrate that subgroups of survivors are at-risk for persistent distress over the course of survivorship, but we also establish that in survivors psychological distress may emerge over several decades following their original cancer diagnosis. These findings have important implications for screening practices among health care providers caring for long-term survivors of childhood cancer.

Overall, the majority of survivors reported no or few symptoms of psychological distress at multiple time points over the 13-year follow-up period. This is consistent with previous cross-sectional reports indicating that elevated distress symptoms may affect only a subset of childhood cancer survivors (Zebrack et al, 2004, 2007; Zeltzer et al, 2008). That the majority of survivors reported no distress over time also supports emerging literature describing the perceived positive impact of cancer in many survivors, consistent with the phenomenon of post-traumatic growth (Zebrack et al, 2012). Moreover, we identified a subset of survivors who reported improvement in distress symptoms over time. Of interest, when compared with survivors who reported similar symptom levels at baseline but did not demonstrate a reduction in symptoms over time, survivors with decreasing symptoms were largely characterised by the absence of risk factors associated with distress (i.e., treatment factors and changes in sociodemographic variables), as opposed to the presence of unique protective factors.

We identified a subset of survivors who reported persistently elevated distress (depression: $8.9 \%$, anxiety: $4.8 \%$, somatisation: $7.2 \%$ ) over the 13-year follow-up period. This finding highlights the potential pervasive course of these symptoms for some childhood cancer survivors and suggests that this group of survivors may benefit from early targeted intervention efforts to reduce distress or better manage physical health symptoms. We previously reported that $19 \%$ of survivors in CCSS initiated treatment with an antidepressant medication over a 10-year followup period (Brinkman et al, 2013); however, the effectiveness of such treatment in this population remains unclear. In fact, we found that initiation of psychoactive medication use in the current sample was associated with increased symptoms of distress over time. Nonpharmacologic approaches toward the management of distress have demonstrated effectiveness in the general population and provide an alternative treatment approach for survivors. Specifically, cognitive-behaviour therapy (CBT) is a well-established treatment for anxiety and depression (Butler et al, 2006), with long-term benefits comparable to or exceeding those observed with pharmacotherapy treatment alone (Shea et al, 1992; DeRubeis et al, 1999; Hart et al, 2012). Of note, we did not have data related to the use of nonpharmacologic treatment approaches in our cohort of survivors.

Importantly, we found a modest proportion of survivors characterised by few distress symptoms at cohort entry that 
Table 3. Multivariable models predicting longitudinal class membership

\begin{tabular}{|l|c|c|c|}
\hline & Anxiety $^{\mathrm{a}}$ & Depression $^{\mathrm{b}}$ & Somatisation $^{\mathrm{c}}$ \\
\hline Class & OR $(95 \% \mathrm{Cl})$ & OR $(95 \% \mathrm{Cl})$ & OR $(95 \% \mathrm{Cl})$ \\
\hline
\end{tabular}

Female sex

\begin{tabular}{|c|c|c|c|}
\hline 1 & 1.0 & - & 1.0 \\
2 & $1.38(1.15-1.67)$ & - & $1.34(1.07-1.69)$ \\
3 & $1.18(0.96-1.45)$ & - & $1.59(1.26-2.00)$ \\
4 & $0.92(0.66-1.27)$ & - & $1.92(1.37-2.70)$
\end{tabular}

Radiation therapy (vs none)

$\mathrm{CRT} \leqslant 20 \mathrm{~Gy}$

\begin{tabular}{|l|c|c|c|}
\hline 1 & - & - & 1.0 \\
2 & - & - & $1.04(0.71-1.52)$ \\
3 & - & - & $1.41(0.97-2.03)$ \\
4 & - & - & $\mathbf{2 . 3 2}(1.33-4.05)$ \\
\hline
\end{tabular}

CRT $>20$ Gy

\begin{tabular}{|c|c|c|c|}
\hline 1 & - & - & 1.0 \\
\hline 2 & - & - & $0.90(0.65-1.23)$ \\
\hline 3 & - & - & $1.16(0.84-1.59)$ \\
\hline 4 & - & - & $1.33(0.81-2.18)$ \\
\hline \multicolumn{4}{|c|}{ Non-cranial radiation } \\
\hline 1 & - & - & 1.0 \\
\hline 2 & - & - & $1.09(0.83-1.42)$ \\
\hline 3 & - & - & $1.14(0.86-1.52)$ \\
\hline 4 & - & - & $2.16(1.41-3.29)$ \\
\hline \multicolumn{4}{|c|}{ Baseline chronic medical condition (vs none) } \\
\hline \multicolumn{4}{|c|}{ Mild/moderate } \\
\hline 1 & 1.0 & 1.0 & 1.0 \\
\hline 2 & $1.67(1.34-2.09)$ & $1.63(1.30-2.03)$ & $1.38(1.05-1.80)$ \\
\hline 3 & $1.01(0.79-1.28)$ & $1.41(1.09-1.83)$ & $1.01(0.78-1.31)$ \\
\hline 4 & $1.62(1.08-2.45)$ & $1.60(1.18-2.16)$ & $1.84(1.19-2.86)$ \\
\hline \multicolumn{4}{|c|}{ Severe/disabling } \\
\hline 1 & 1.0 & 1.0 & 1.0 \\
\hline 2 & $1.63(1.28-2.06)$ & $1.46(1.15-1.85)$ & $1.32(0.99-1.77)$ \\
\hline 3 & $0.76(0.58-0.99)$ & $0.86(0.64-1.16)$ & $0.77(0.57-1.04)$ \\
\hline 4 & $1.30(0.84-2.02)$ & $1.25(0.90-1.72)$ & $2.03(1.30-3.18)$ \\
\hline
\end{tabular}

Physical health status change (vs no change)

Improved health status

\begin{tabular}{|l|c|c|c|}
\hline 1 & 1.0 & 1.0 & 1.0 \\
2 & $1.72(0.94-3.12)$ & $0.71(0.38-1.29)$ & $1.02(0.50-2.10)$ \\
3 & $0.80(0.40-1.58)$ & $0.39(0.18-0.83)$ & $0.29(0.12-0.72)$ \\
4 & $0.72(0.34-1.52)$ & $0.63(0.32-1.22)$ & $0.44(0.20-0.93)$ \\
\hline
\end{tabular}

Worsened health status

\begin{tabular}{|c|c|c|c|}
\hline 1 & 1.0 & 1.0 & 1.0 \\
\hline 2 & $1.07(0.75-1.51)$ & $1.23(0.86-1.76)$ & $1.18(0.71-1.94)$ \\
\hline 3 & $2.96(2.22-3.96)$ & $3.30(2.41-4.51)$ & $5.33(3.86-7.36)$ \\
\hline 4 & $3.43(2.25-5.24)$ & $2.89(2.04-4.08)$ & $4.35(2.77-6.81)$ \\
\hline \multicolumn{4}{|c|}{ Cancer-related pain change (vs no change) } \\
\hline \multicolumn{4}{|c|}{ Improved pain } \\
\hline 1 & - & 1.0 & 1.0 \\
\hline 2 & - & $0.68(0.35-1.31)$ & $1.19(0.60-2.37)$ \\
\hline 3 & - & $1.10(0.53-2.25)$ & $0.82(0.40-1.71)$ \\
\hline 4 & - & $0.96(0.51-1.78)$ & $0.45(0.22-0.95)$ \\
\hline
\end{tabular}

\section{Table 3. (Continued)}

\begin{tabular}{|l|c|c|c|}
\hline & Anxiety $^{\mathrm{a}}$ & Depression $^{\mathrm{b}}$ & Somatisation $^{\mathrm{c}}$ \\
\hline Class & OR $(95 \% \mathrm{Cl})$ & OR $(95 \% \mathrm{Cl})$ & OR $(95 \% \mathrm{Cl})$ \\
\hline
\end{tabular}

\section{Worsened pain}

\begin{tabular}{|l|l|c|c|}
\hline 1 & - & 1.0 & 1.0 \\
2 & - & $1.14(0.77-1.69)$ & $\mathbf{1 . 8 5}(\mathbf{1 . 1 7 - 2 . 9 5 )}$ \\
3 & - & $1.01(0.65-1.57)$ & $\mathbf{2 . 3 8}(\mathbf{1 . 5 8 - 3 . 5 8 )}$ \\
4 & - & $\mathbf{2 . 1 3 ( 1 . 4 3 - 3 . 1 9 )}$ & $\mathbf{3 . 3 2}(\mathbf{2 . 0 2 - 5 . 4 3 )}$ \\
\hline
\end{tabular}

Employment change (vs no change)

Reduced employment

\begin{tabular}{|l|c|c|c|}
\hline 1 & - & - & 1.0 \\
2 & - & - & $0.86(0.59-1.25)$ \\
3 & - & - & $1.42(1.04-1.94)$ \\
4 & - & - & $1.84(1.22-2.79)$ \\
\hline
\end{tabular}

Acquired employment

\begin{tabular}{|l|c|c|c|}
\hline 1 & - & - & 1.0 \\
2 & - & - & $1.89(0.77-4.67)$ \\
3 & - & - & $0.80(0.34-1.89)$ \\
4 & - & - & $0.94(0.38-2.36)$
\end{tabular}

Personal income change (vs no change)

\section{Decreased income}

\begin{tabular}{|l|c|c|c|}
\hline 1 & - & - & 1.0 \\
2 & - & - & $0.27(0.11-0.65)$ \\
3 & - & - & $0.53(0.29-0.94)$ \\
4 & - & - & $0.78(0.40-1.53)$ \\
\hline
\end{tabular}

Increased income

\begin{tabular}{|l|c|c|c|}
\hline 1 & - & - & 1.0 \\
2 & - & - & $1.18(0.93-1.48)$ \\
3 & - & - & $0.97(0.76-1.24)$ \\
4 & - & - & $1.35(0.93-1.94)$ \\
\hline
\end{tabular}

Marital status change (vs no change)

Single to married

\begin{tabular}{|l|l|l|l|}
\hline 1 & - & 1.0 & - \\
2 & - & $1.43(1.11-1.84)$ & - \\
3 & - & $0.67(0.48-0.92)$ & - \\
4 & - & $0.36(0.25-0.51)$ & - \\
\hline
\end{tabular}

Married to single

\begin{tabular}{|l|l|c|c|}
\hline 1 & - & 1.0 & - \\
2 & - & $0.92(0.45-1.86)$ & - \\
3 & - & $0.94(0.39-2.23)$ & - \\
4 & - & $2.25(1.10-4.60)$ & - \\
\hline
\end{tabular}

Antidepressant change (vs no change)

Initiated use

\begin{tabular}{|l|c|c|c|}
\hline 1 & 1.0 & 1.0 & 1.0 \\
2 & $1.58(1.23-2.03)$ & $1.18(0.89-1.55)$ & $1.22(0.89-1.66)$ \\
3 & $3.01(2.35-3.85)$ & $\mathbf{2 . 9 2 ( 2 . 2 5 - 3 . 7 8 )}$ & $1.38(1.03-1.85)$ \\
4 & $1.89(1.26-2.84)$ & $\mathbf{2 . 7 2 ( 2 . 0 2 - 3 . 6 5 )}$ & $1.46(0.99-2.16)$ \\
\hline
\end{tabular}

\section{Stopped use}

\begin{tabular}{l|c|c|c|}
1 & 1.0 & 1.0 & 1.0 \\
2 & $0.57(0.26-1.24)$ & $0.84(0.39-1.79)$ & $2.21(0.86-5.65)$ \\
3 & $0.78(0.33-1.87)$ & $0.57(0.19-1.72)$ & $0.71(0.24-2.09)$ \\
4 & $0.28(0.11-0.77)$ & $0.33(0.14-0.82)$ & $0.73(0.25-2.09)$ \\
\hline
\end{tabular}




\begin{tabular}{|c|c|c|c|}
\hline & Anxiety ${ }^{a}$ & Depression $^{b}$ & Somatisation $^{c}$ \\
\hline Class & OR $(95 \% \mathrm{Cl})$ & OR $(95 \% \mathrm{Cl})$ & OR $(95 \% \mathrm{Cl})$ \\
\hline \multicolumn{4}{|c|}{ Anxiolytic change (vs no change) } \\
\hline \multicolumn{4}{|c|}{ Initiated use } \\
\hline $\begin{array}{l}1 \\
2 \\
3 \\
4\end{array}$ & $\begin{array}{c}1.0 \\
0.88(0.53-1.45) \\
2.77(1.90-4.04) \\
4.11(2.52-6.72)\end{array}$ & $\begin{array}{c}1.0 \\
0.92(0.56-1.50) \\
2.10(1.41-3.24) \\
1.60(1.00-2.56)\end{array}$ & $\begin{array}{l}- \\
- \\
-\end{array}$ \\
\hline \multicolumn{4}{|c|}{ Stopped use } \\
\hline $\begin{array}{l}1 \\
2 \\
3 \\
4\end{array}$ & $\begin{array}{c}1.0 \\
1.07(0.40-2.87) \\
0.95(0.28-3.21) \\
0.52(.016-1.64)\end{array}$ & $\begin{array}{c}1.0 \\
0.72(0.26-2.00) \\
0.64(0.20-2.01) \\
0.90(0.28-2.87)\end{array}$ & $\begin{array}{l}- \\
- \\
-\end{array}$ \\
\hline \multicolumn{4}{|c|}{ Analgesic change (vs no change) } \\
\hline \multicolumn{4}{|c|}{ Initiated use } \\
\hline $\begin{array}{l}1 \\
2 \\
3 \\
4\end{array}$ & $\begin{array}{c}1.0 \\
1.19(0.78-1.80) \\
1.37(0.92-2.05) \\
1.59(0.90-2.80)\end{array}$ & $\begin{array}{l}- \\
- \\
- \\
-\end{array}$ & $\begin{array}{c}1.0 \\
0.69(0.36-1.33) \\
1.95(1.28-2.96) \\
2.01(1.16-3.51)\end{array}$ \\
\hline \multicolumn{4}{|c|}{ Stopped use } \\
\hline $\begin{array}{l}1 \\
2 \\
3 \\
4\end{array}$ & $\begin{array}{c}1.0 \\
1.45(1.13-1.87) \\
0.94(0.68-1.30) \\
2.02(1.36-2.99)\end{array}$ & $\begin{array}{l}- \\
- \\
-\end{array}$ & $\begin{array}{c}1.0 \\
1.89(1.40-2.53) \\
1.07(0.75-1.52) \\
2.30(1.56-3.39)\end{array}$ \\
\hline \multicolumn{4}{|c|}{$\begin{array}{l}\text { Abbreviations: } \mathrm{Cl}=\text { confidence interval; } \mathrm{CRT}=\text { cranial radiation therapy; } \mathrm{OR}=\text { odds ratio; } \\
-=\text { not selected in best-fitting model. Bold font denotes statistical significance. } \\
\mathrm{a}_{\text {Model adjusted for baseline antidepressant use, anxiolytic use, employment status, }} \\
\text { cancer-related pain and physical health status. } \\
\mathrm{b}_{\text {Model adjusted for baseline antidepressant use, anxiolytic use, marital status, insurance, }} \\
\text { personal income, cancer-related pain and physical health status. } \\
{ }^{\mathrm{M}} \text { Model adjusted for antidepressant use, anxiolytic use, education level, employment status, } \\
\text { cancer-related pain and physical health status. }\end{array}$} \\
\hline
\end{tabular}

increased steadily over time (depression: $10.2 \%$, anxiety: $11.8 \%$, somatisation: $13.0 \%)$. This pattern reflects a group of survivors previously undetected in cross-sectional follow-up studies of childhood cancer survivors. Moreover, this group represents survivors who would not be identified through a single screening of distress at the time of study cohort entry. Thus, these data highlight the need for regular, repeated screenings of psychological distress in adult survivors of childhood cancer. Such screenings may be particularly important for survivors who develop late medical morbidities that result in reduced health status.

Notably, we found that survivor perception of worsened physical health status and increased pain was significantly associated with reports of persistent and increasing symptoms of distress over time. Moreover, changes in socioeconomic factors such as marriage and employment were associated with persistent and increasing distress. The presence of psychological distress may be conceptualised within a biopsychosocial model of health, which holds that health and illness result from the complex interplay of biological, psychological, and social factors (Engel, 1981). Thereby, macrolevel processes such as social support (e.g., marriage, employment) and microlevel processes (e.g. chemical imbalances, organ dysfunction) interact to produce a state of health or illness.

Effective distress management requires timely and accurate identification of symptoms. As only a small proportion of adult survivors of childhood cancer are followed by an oncologist (Oeffinger et al, 2004), the burden of identifying distress symptoms
Table 4. Multivariable models predicting decreasing distress over time

\begin{tabular}{|l|c|l|r|}
\hline & Anxiety $^{\mathrm{a}}$ & Depression $^{\mathrm{b}}$ & Somatisation $^{\mathrm{c}}$ \\
\hline Class & OR $(95 \% \mathrm{Cl})$ & OR $(95 \% \mathrm{Cl})$ & OR $(95 \% \mathrm{Cl})$ \\
\hline
\end{tabular}

Female sex

\begin{tabular}{|l|l|c|c}
\hline 2 & - & $1.57(1.16-2.13)$ & - \\
4 & - & 1.0 & - \\
\hline
\end{tabular}

Radiation therapy (vs none)

$\mathrm{CRT} \leqslant 20 \mathrm{~Gy}$

\begin{tabular}{|l|c|c|c|}
\hline 2 & - & - & $0.66(0.35-1.24)$ \\
4 & - & - & 1.0 \\
\hline \multicolumn{4}{|l|}{ CRT > 20 Gy } \\
\hline 2 & - & - & $0.82(0.48-1.40)$ \\
4 & - & - & 1.0
\end{tabular}

Non-cranial radiation

\begin{tabular}{|l|c|c|c|}
\hline 2 & - & - & $0.57(0.3-0.91)$ \\
4 & - & - & 1.0 \\
\hline
\end{tabular}

Baseline chronic medical condition (vs none)

\section{Mild/moderate}

\begin{tabular}{|l|c|c|c|}
\hline 2 & - & - & $0.77(0.47-1.26)$ \\
4 & - & - & 1.0 \\
\hline \multicolumn{4}{|l|}{ Severe/disabling } \\
\hline 2 & - & - & $0.64(0.39-1.06)$ \\
4 & - & - & 1.0
\end{tabular}

Physical health status change (vs no change)

Improved health status

\begin{tabular}{|l|c|c|c|}
\hline 2 & $1.15(0.60-2.19)$ & $0.90(0.53-1.52)$ & $1.90(0.86-4.20)$ \\
4 & 1.0 & 1.0 & 1.0 \\
\hline
\end{tabular}

Worsened health status

\begin{tabular}{|l|c|c|c|}
\hline 2 & $0.37(0.22-0.62)$ & $0.36(0.23-0.57)$ & $0.30(0.17-0.54)$ \\
4 & 1.0 & 1.0 & 1.0 \\
\hline
\end{tabular}

Cancer-related pain change (vs no change)

Improved pain

\begin{tabular}{|l|c|c|c|}
\hline 2 & $1.69(0.74-3.85)$ & - & $1.97(0.89-4.34)$ \\
4 & 1.0 & - & 1.0 \\
\hline
\end{tabular}

Worsened pain

\begin{tabular}{|l|c|c|c|}
\hline 2 & $0.53(0.29-0.96)$ & - & $0.67(0.38-1.20)$ \\
4 & 1.0 & - & 1.0 \\
\hline
\end{tabular}

\begin{tabular}{l|c|c|c}
\hline 4 & 1.0 & - & 1.0
\end{tabular}

Employment change (vs no change)

Reduced employment

\begin{tabular}{|l|c|c|c|}
\hline 2 & - & $0.67(0.45-0.98)$ & $0.33(0.21-0.54)$ \\
4 & - & 1.0 & 1.0 \\
\hline
\end{tabular}

Acquired employment

\begin{tabular}{|l|c|c|c|}
\hline 2 & - & $0.65(0.32-1.29)$ & $2.30(0.82-6.47)$ \\
4 & - & 1.0 & 1.0 \\
\hline
\end{tabular}


Table 4. (Continued)

\begin{tabular}{|l|c|c|c|}
\hline & Anxiety $^{\mathrm{a}}$ & Depression $^{\mathrm{b}}$ & Somatisation $^{\mathrm{c}}$ \\
\hline Class & OR $(95 \% \mathrm{Cl})$ & OR $(95 \% \mathrm{Cl})$ & OR $(95 \% \mathrm{Cl})$ \\
\hline
\end{tabular}

Personal income change (vs no change)

\section{Decreased income}

\begin{tabular}{|l|c|c|c|}
\hline 2 & - & - & - \\
4 & - & - & - \\
\hline \multicolumn{4}{|l|}{ Increased income } \\
\hline 2 & - & - & - \\
4 & - & - & - \\
\hline
\end{tabular}

Marital status change (vs no change)

Single to married

\begin{tabular}{|l|c|c|c|}
\hline 2 & $1.60(1.01-2.54)$ & $2.73(1.92-3.89)$ & - \\
4 & 1.0 & 1.0 & - \\
\hline \multicolumn{4}{|l|}{ Married to single } \\
\hline 2 & $1.58(0.45-5.53)$ & $0.82(0.33-2.02)$ & - \\
4 & 1.0 & 1.0 & - \\
\hline
\end{tabular}

Antidepressant change (vs no change)

Initiated use

\begin{tabular}{|c|c|c|c|}
\hline $\begin{array}{l}2 \\
4\end{array}$ & - & $\begin{array}{c}0.37(0.26-0.55) \\
1.0\end{array}$ & - \\
\hline \multicolumn{4}{|c|}{ Stopped use } \\
\hline 2 & - & $2.31(0.94-5.68)$ & - \\
\hline 4 & - & 1.0 & - \\
\hline
\end{tabular}

Anxiolytic change (vs no change)

Initiated use

\begin{tabular}{|l|c|c|c|}
\hline 2 & $0.18(0.10-0.33)$ & - & $0.44(0.21-0.94)$ \\
4 & 1.0 & - & 1.0 \\
\hline \multicolumn{4}{|l|}{ Stopped use } \\
\hline 2 & $2.27(0.68-7.52)$ & - & $0.63(0.28-1.43)$ \\
4 & 1.0 & - & 1.0 \\
\hline
\end{tabular}

Analgesic change (vs no change)

Initiated use
\begin{tabular}{|l|c|c|c|}
\hline 2 & - & - & $0.35(0.16-0.78)$ \\
4 & - & - & 1.0 \\
\hline
\end{tabular}

Stopped use
\begin{tabular}{|l|c|c|c|} 
\\
2 & - & - & $0.84(0.54-1.29)$ \\
4 & - & - & 1.0
\end{tabular}

Abbreviations: $\mathrm{Cl}=$ confidence interval; $\mathrm{CRT}=$ cranial radiation therapy; $\mathrm{OR}=$ odds ratio $-=$ not selected in best fitting model. Bold font denotes statistical significance.

${ }^{a}$ Model adjusted for baseline psychoactive medication use, employment status and cancer-

related pain.

Model adjusted for baseline antidepressant use and cancer-related pain

${ }^{\mathrm{c}}$ Model adjusted for baseline employment, cancer-related pain and physical health status. in this survivor population often falls to general practitioners. Previous data suggest that most physicians working with oncology patients do not feel confident dealing with distress and few utilise standardised questionnaires to assess patient distress (Mitchell et al, 2008). Moreover, front-line clinicians demonstrate approximately $50 \%$ sensitivity and $80 \%$ specificity when assessing distress in cancer patients (Mitchell et al, 2011). These data, in the context of our findings, suggest that routine screening of psychological distress with a validated instrument (e.g., Brief Symptom Inventory) is warranted in adult survivors of childhood cancer. However, comprehensive care extends beyond screening and requires appropriate referrals for mental health evaluations and indicated treatment (e.g., CBT).

Although our study provides important insights toward understanding the trajectory of psychological distress in adult survivors of childhood cancer, we must acknowledge several limitations. The longitudinal analysis only included data from survivors who completed the BSI-18 at all three study time points. Although survivors who completed the BSI-18 at one or two time points did not differ from those who completed all three on key demographic and treatment factors at baseline, we cannot rule out the potential influence of selection bias. The BSI-18 provides a measure of distress symptoms over the previous 7 days. Although our study was longitudinal, the infrequent measurement of distress coupled with the short symptom reporting window, may result in an inaccurate estimation of survivors who experienced distress symptoms over the 13-year follow-up. Our study used, almost entirely, self-reported data to measure outcomes and predictor variables. We did not consider reciprocal relationships between predictors and outcomes. It is possible that distress levels influenced reporting of certain factors, such as perceived health status and pain. In addition, there are likely several unmeasured variables that may contribute to distress symptoms in this population of survivors. Future research is needed to examine comorbidities between types of distress and between distress and other factors such as fatigue, personality, social support and community integration. Finally, although we identified subgroups of survivors with divergent patterns of distress symptoms over time, age and time since diagnosis were fairly heterogeneous both within and across study time points. This limits our ability to generalise our findings to other groups of adult survivors of childhood cancer.

Our results show that well into adult survivorship, the majority of childhood cancer survivors do not present with elevated distress symptoms. However, we identified two subgroups of survivors who will likely benefit from intervention to mitigate or prevent the onset of symptoms: (1) survivors with persistently elevated distress and (2) survivors with emerging distress symptoms over time. Toward this goal, our data underscore the importance of routine screening of psychological morbidities in adult survivors of childhood cancer.

\section{ACKNOWLEDGEMENTS}

This work was supported by grant U24 CA 055727 (LLR) from the National Cancer Institute, with additional support provided to St Jude Children's Research Hospital by the Cancer Center Support (CORE) grant CA21765 and by ALSAC.

\section{REFERENCES}

Brinkman TM, Ullrich NJ, Zhang N, Green DM, Zeltzer LK, Lommel KM, Brouwers P, Srivastava DK, Jain N, Robison LL, Krull KR (2013)

Prevalence and predictors of prescription psychoactive medication use in 
adult survivors of childhood cancer: a report from the Childhood Cancer Survivor Study. J Cancer Surviv 7(1): 104-114.

Butler AC, Chapman JE, Forman EM, Beck AT (2006) The empirical status of cognitive- behavioral therapy: a review of meta-analyses. Clin Psychol Rev 26(1): 17-31.

Cancer Therapy Evaluation Program. Common Terminology Criteria for Adverse Events, version 3.0. National Cancer Institute: Bethesda, MD, 2003. Available at http://ctep.cancer.gov/protocolDevelopment/electronic_ applications/docs/ctcaev3.pdf (accessed 10 April 2013).

Derogatis L (2000) Brief Symptom Inventory (BSI): Administration, Scoring, and Procedures Manual. NCS Pearson: Minneapolis, MN, USA.

DeRubeis RJ, Gelfand LA, Tang TZ, Simons AD (1999) Medications versus cognitive behavior therapy for severely depressed outpatients: mega-analysis of four randomized comparisons. Am J Psychiatry 156(7): 1007-1013.

Engel GL (1981) The clinical application of the biopsychosocial model. J Med Philos 6: 101-123.

Hart SL, Hoyt MA, Diefenbach M, Anderson DR, Kilbourn KM, Craft LL, Steel JL, Cuijpers P, Mohr DC, Berendsen M, Spring B, Stanton AL (2012) Meta-analysis of efficacy of interventions for elevated depressive symptoms in adults diagnosed with cancer. J Natl Cancer Inst 104(13): 990-1004

Hope S, Rodgers B, Power C (1999) Marital status transitions and psychological distress: longitudinal evidence from a national population sample. Psychol Med 29(2): 381-389.

Howlader N, Noone A, Krapcho M, Neyman N, Aminou R, Waldron W (2011) SEER Cancer Statistics Review, 1975-2008. Available at http://seer.cancer.gov/csr/1975_2008/.

Hudson MM, Mertens AC, Yasui Y, Hobbie W, Chen H, Gurney JG, Yeazel M, Recklitis CJ, Marina N, Robison LR, Oeffinger KC. Childhood Cancer Survivor Study I (2003) Health status of adult long-term survivors of childhood cancer: a report from the Childhood Cancer Survivor Study. JAMA 290(12): 1583-1592.

Leisenring WM, Mertens AC, Armstrong GT, Stovall MA, Neglia JP, Lanctot JQ, Boice JD, Whitton JA, Yasui Y (2009) Pediatric cancer survivorship research: experience of the Childhood Cancer Survivor Study. J Clin Oncol 27(14): 2319-2327.

Michel G, Rebholz CE, von der Weid NX, Bergstraesser E, Kuehni CE (2010) Psychological distress in adult survivors of childhood cancer: the Swiss Childhood Cancer Survivor study. J Clin Oncol 28(10): 1740-1748.

Miech R, Power C, Eaton WW (2007) Disparities in psychological distress across education and sex: a longitudinal analysis of their persistence within a cohort over 19 years. Ann Epidemiol 17(4): 289-295.

Miech R, Shanahan MJ (2000) Socioeconomic status and depression over the life course. J Health Soc Behav 41: 162-176.

Mitchell AJ, Hussain N, Grainger L, Symonds P (2011) Identification of patient-reported distress by clinical nurse specialists in routine oncology practice: a multicentre UK study. Psychooncology 20(10): 1076-1083.

Mitchell AJ, Kaar S, Coggan C, Herdman J (2008) Acceptability of common screening methods used to detect distress and related mood disorderspreferences of cancer specialists and non-specialists. Psychooncology 17(3): 226-236.

Muthén LK, Muthén BO (1998-2010) Mplus User's Guide, 6th edn. Muthén \& Muthén: Los Angeles, CA.

Oeffinger KC, Mertens AC, Sklar CA, Kawashima T, Hudson MM, Meadows AT, Friedman DL, Marina N, Hobbie W, Kadan-Lottick NS, Schwartz CL, Leisenring W, Robison LL. Childhood Cancer Survivor S (2006) Chronic health conditions in adult survivors of childhood cancer. $N$ Engl J Med 355(15): 1572-1582.

Oeffinger KC, Mertens AC, Hudson MM, Gurney JG, Casillas J, Chen H, Whitton J, Yeazel M, Yasui Y, Robison LL (2004) Health care of young adult survivors of childhood cancer: a report from the Childhood Cancer Survivor Study. Ann Fam Med 2(1): 61-70.

Recklitis CJ, Diller LR, Li X, Najita J, Robison LL, Zeltzer L (2010) Suicide ideation in adult survivors of childhood cancer: a report from the Childhood Cancer Survivor Study. J Clin Oncol 28(4): 655-661.

Recklitis CJ, Lockwood RA, Rothwell MA, Diller LR (2006a) Suicidal ideation and attempts in adult survivors of childhood cancer. J Clin Oncol 24(24): 3852-3857.

Recklitis CJ, Parsons SK, Shih MC, Mertens A, Robison LL, Zeltzer L (2006b) Factor structure of the brief symptom inventory-18 in adult survivors of childhood cancer: results from the childhood cancer survivor study. Psychol Assess 18(1): 22-32.

Recklitis CJ, Rodriguez P (2007) Screening childhood cancer survivors with the brief symptom inventory-18: classification agreement with the symptom checklist-90-revised. Psychooncology 16(5): 429-436.

Robison LL, Armstrong GT, Boice JD, Chow EJ, Davies SM, Donaldson SS, Green DM, Hammond S, Meadows AT, Mertens AC, Mulvihill JJ, Natan PC, Neglia JP, Pecker RJ, Rajaraman P, Asklar C, Stovall M, Strong LC, Yasui Y, Zeltzer LK (2009) The Childhood Cancer Survivor Study: a National Cancer Institute-supported resource for outcome and intervention research. J Clin Oncol 27(14): 2308-2318.

Schieman S, Van Gundy K, Taylor J (2001) Status, role, and resource explanations for age patterns in psychological distress. J Health Soc Behav 42(1): 80-96.

Shea MT, Elkin I, Imber SD, Sotsky SM, Watkins JT, Collins JF, Pilkonis PA, Beckham E, Glass DR, Dolan RT, Morris B Parloff, Boice Jr JJ, Whitton JA, Yasui Y (1992) Course of depressive symptoms over follow-up. Findings from the National Institute of Mental Health Treatment of Depression Collaborative Research Program. Arch Gen Psychiatry 49(10): 782-787.

Trotti A, Colevas AD, Setser A, Rusch V, Jaques D, Budach V, Langer C, Murphy B, Cumberlin R, Coleman CN, Rubin P (2003) CTCAE v3.0: development of a comprehensive grading system for the adverse effects of cancer treatment. Semin Radiat Oncol 13(3): 176-181.

Zebrack BJ, Gurney JG, Oeffinger K, Whitton J, Packer RJ, Mertens A, Turk N, Castleberry R, Dreyer Z, Robison LL, Zeltzer LK (2004) Psychological outcomes in long-term survivors of childhood brain cancer: a report from the childhood cancer survivor study. J Clin Oncol 22(6): 999-1006.

Zebrack BJ, Stuber ML, Meeske KA, Phipps S, Krull KR, Liu Q, Yasui Y, Parry C, Hamilton R, Robison LL, Zeltzer LK (2012) Perceived positive impact of cancer among long-term survivors of childhood cancer: a report from the childhood cancer survivor study. Psychooncology 21(6): 630-639.

Zebrack BJ, Zevon MA, Turk N, Nagarajan R, Whitton J, Robison LL, Zeltzer LK (2007) Psychological distress in long-term survivors of solid tumors diagnosed in childhood: a report from the childhood cancer survivor study. Pediatr Blood Cancer 49(1): 47-51.

Zeltzer LK, Lu Q, Leisenring W, Tsao JC, Recklitis C, Armstrong G, Mertens AC, Robison LL, Ness KK (2008) Psychosocial outcomes and health-related quality of life in adult childhood cancer survivors: a report from the childhood cancer survivor study. Cancer Epidemiol Biomarkers Prev 17(2): $435-446$.

This work is published under the standard license to publish agreement. After 12 months the work will become freely available and the license terms will switch to a Creative Commons AttributionNonCommercial-Share Alike 3.0 Unported License.

Supplementary Information accompanies this paper on British Journal of Cancer website (http://www.nature.com/bjc) 